\title{
PROBABILITY OF TRAUMATIC SITUATIONS IN MECHANIZED PROCESSES IN AGRICULTURE USING MATHEMATICAL APPARATUS OF MARKOV CHAIN METHOD
}

\author{
Oleksandr Voinalovych ${ }^{1}$, Oleg Hnatiuk ${ }^{1}$, Ivan Rogovskii ${ }^{1}$, Oleksandr Pokutnii ${ }^{2}$ \\ ${ }^{1}$ National University of Life and Environmental Sciences of Ukraine, Ukraine; ${ }^{2}$ Institute of \\ Mathematics of National Academy of Sciences of Ukraine, Ukraine \\ voynalov@bigmir.net, olegnatyk@ukr.net,rogovskii@nubip.edu.ua, alex_poker@imath.kiev.ua
}

\begin{abstract}
In this paper for exploring the mechanisms of formation of traumatic situations, the conditions and circumstances that contribute to them, and the study of the course of events leading to dangers in mechanized processes in agriculture the mathematical apparatus was used developed by the probability theory for Markov random processes with discrete states and continuous time, when the transition of a system from one state to another is possible at any unknown random time. It has been established that the processes of traumatic situations and their consequences can be represented by graph structures, using four states of the "man-machineindustrial environment" system: working and defective conditions of the tractor (machinery), hit of the machine operator into a dangerous and emergency condition (situation). A system of Kolmogorov differential equations was made, in which unknown functions are the probabilities of states of the system as functions of time with the normative condition that the sum of these probabilities will be equal to 1 . Introducing the matrix of the intensity of events, the Laplace transform was used to solve the system of differential equations, which allowed the system to be transformed into a linear algebraic system. Elements of the matrix of intensities of the flows of events that bind separate states of the graph were given taking into account the probabilities of accidents with mechanics, established on the basis of the statistics of occupational injuries and data of accumulation of cracks in the array of tractor parts, were determined as a result of defectoscopic control. The results of the research allow in the medium and long term perspective to predict the probable states of the "human-machine-industrial environment" system in the risks of professional injury of agricultural machine operators.
\end{abstract}

Keywords: tractor, risk, operator, traumatic situation, defectoscopic control.

\section{Introduction}

The system of introduction of technical means of safety and preventive measures for prevention of occupational injuries of agricultural workers should be based on an analysis of its causes through the improvement of available methods of probabilistic assessment of the risk of emergencies, taking into account the specific features of mechanized processes in the agro-industrial complex. Therefore, the study of the mechanism of formation of traumatic situations, the conditions and circumstances that contribute to them, the study of the nature of the course of events that lead to the dangers of mechanized processes in the agro-industrial complex, is now a topical task. It is necessary to switch from the qualitative methods for analyzing the causes of occupational injuries on the basis of expert assessments to the quantitative methods for assessing the probability of occurrence of emergencies involving mobile agricultural machinery, which should take into account the degree of its serviceability.

Machine-tractor aggregates (MTA), machine operators - operators of mobile agricultural machinery and parameters of the production environment when performing mechanized works in agriculture should be considered as interconnected elements of a holistic system [1]. According to some researchers, the weak link of such a system can be workers with their false actions and violations of the labor safety requirements, other researchers justify the high level of traumatism of machine operators by the influence of parameters of the production environment [2]. The common understanding is that the change in the state of any of the elements of the system machine - man production environment (M-M-PE) necessarily leads to a change in the state of the system as a whole [3]. However, the important role of the technical condition of the machine and the functionality of the safety equipment set up on it in the formation of traumatic situations is often left out of focus [4].

Changes in the system of M-M-PE by their nature are considered in a separate manner: as gradual, for example, accumulation of scattered operational damage in an array of details of the machine nodes or accumulation of employee weariness during the change and suddenly occurring at certain moments of time (detachment of parts, loss concentration of the operator or critical manifestation of environmental parameters) [5]. However, the gradual accumulation of operational defects in the part (element of the design) eventually leads to the emergence and spread of a major crack of critical size, 
which causes the sudden failure of the machine node (unit), and therefore the onset of a dangerous situation [6].

For the analysis of sudden changes in the M-M-PE system, a mathematical apparatus developed in the theory of probabilities for Markov random processes with discrete states and continuous time is used when there is transition of a system from one state to another in any unknown, random, instantaneous time $t$ [7]. However, in the developed mathematical models the transition from a system to a faulty state is viewed primarily as a consequence of the false actions of the machine operator (or other employee), without analyzing the degree of accumulation of operational defects in an array of the parts of the unit, which may be determinative for creation of an emergency (traumatic) situation.

\section{Materials and methods}

In this paper, the method of calculation of professional risks of mechanizers is proposed on the basis of the analysis of the graph of the states of the processes of traumatic situations during the operation of MTA [8].

According to the methodology [9], random processes with discrete states and continuous time are considered, and the transitions of the system $S$ from one state to another are described as occurring under the influence of certain event flows. Then the probability density of the transition $\lambda$ gets the meaning of the intensities (densities) of the corresponding event flows. If all these flows are Poisson (ordinary and without an aftereffect, with constant or time-dependent intensity), then the process occurring in the system $S$ will be Markovian.

The degree of deterioration of the technical condition of tractors after prolonged exploitation in the work [7] was estimated according to the data on the intensity of accumulation of defects (cracks) in the array of tractor parts detected during the defectoscopic control of the responsible parts (construction elements) of the tractors. In the used vibration current defectoscope it was possible to switch the sensitivity to the degree of defects of different sizes [4], which allowed the total number of identified operational defects to distinguish small-sized (on the threshold of the sensitivity of the defectoscope) that at the moment did not pose a threat to safe operation of the tractor.

\section{Results and discussion}

To analyze the traumatic situation that may occur on mechanized processes in the agroindustrial complex a graph of the states was presented in Fig. 1.

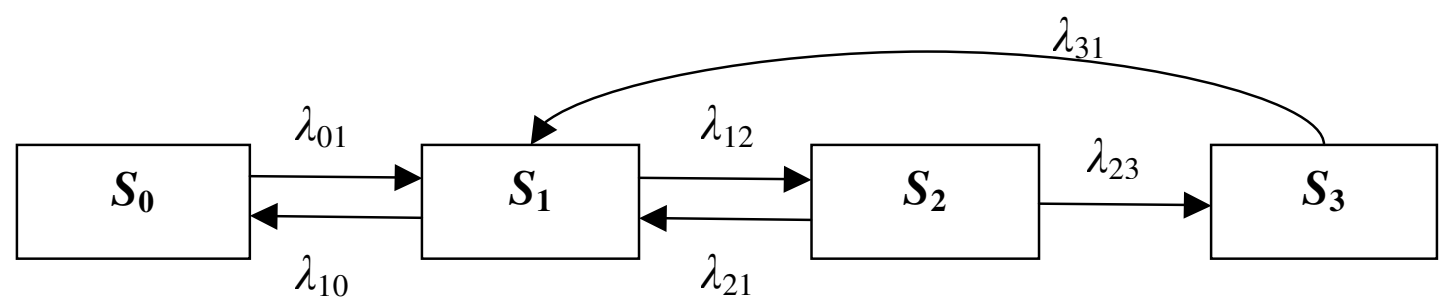

Fig. 1. Graph of states of process of traumatic situation: $S_{0}$ - system is in the initial (proper) state; $S_{1}$ - system is in a working conditionally conditional state; $S_{2}$ - system is in a faulty state; $S_{3}$ - the state of the system is dangerous for workers servicing the agricultural unit

At the beginning of the MTA operation, the system is in a good condition $S_{0}$, and after a certain period of time, from the effects of events with intensity $\lambda_{01}$, the state of the system deteriorates, however, it does not affect the system's robustness for some time and it can continue to function. Such a state can be characterized as a working (conditionally sound) state $S_{1}$. Being in the state of $S_{1}$, the system can return after a certain time to the state $S_{0}$ from the action of events with intensity $\lambda_{10}$. On the other hand, from the effects of event fluctuations with intensity $\lambda_{12}$, for example, due to noncompliance by the mechanician of the scheduled technical inspection or distribution of truncated cracks in the details of the nodes (structural elements), the system can go into a faulty state $S_{2}$.

Consider the following options for development of the event:

1. from the effects of event streams with intensity $\lambda_{21}$ (for example, after repair of a certain node of a tractor or replacement of defective parts), the system returns to the state $S_{1}$, which may or may not 
continue, or subsequently, from the effects of events with intensity of $\lambda_{10}$, may return to the state $S_{0}$;

2. from the events with intensity $\lambda_{23}$ the system passes to the dangerous state $S_{3}$ with possible consequences in the form of injury to the machine operator or returns to the state of $S_{1}$ from the effects of events with intensity $\lambda_{31}$ (for example, due to capital repairs of MTA).

Having analyzed the graph of the states in Fig. 1, we determine the probabilities of the states of the analyzed system $P_{0}(t), P_{1}(t), P_{2}(t)$ and $P_{3}(t)$ as a function of time. These probabilities must satisfy the system of the differential Kolmogorov equations, with probabilities of states of the system as unknown functions (1):

$$
\left\{\begin{array}{l}
\frac{d P_{0}}{d t}=-P_{0} \lambda_{01}+P_{1} \lambda_{10} \\
\frac{d P_{1}}{d t}=-P_{1} \lambda_{10}-P_{1} \lambda_{12}+P_{0} \lambda_{01}+P_{2} \lambda_{21}+P_{3} \lambda_{31} \\
\frac{d P_{2}}{d t}=-P_{2} \lambda_{21}-P_{2} \lambda_{23}+P_{1} \lambda_{12}, \\
\frac{d P_{3}}{d t}=-P_{3} \lambda_{31}+P_{2} \lambda_{23}
\end{array}\right.
$$

where $P_{0}+P_{1}+P_{2}+P_{3}=1-$ normalization condition.

If we consider the vector-function $P(t)=\left(P_{0}(t) ; P_{1}(t) ; P_{2}(t) ; P_{3}(t)\right)$ and the intensity matrix, then the system of Kolmogorov equations will have the form of a linear matrix system of differential equations (2):

$$
\Lambda=\left(\begin{array}{llll}
-\lambda_{01} & \lambda_{10} & 0 & 0 \\
\lambda_{01} & -\lambda_{10}-\lambda_{12} & \lambda_{21} & \lambda_{31} \\
0 & \lambda_{12} & -\lambda_{21}-\lambda_{23} & 0 \\
0 & 0 & \lambda_{23} & -\lambda_{31}
\end{array}\right) .
$$

After solving this system of differential equations using the Laplace transform, one can find the probabilities of the states of the analyzed system (3).

$$
\left\{\begin{array}{c}
\frac{d \vec{P}(t)}{d t}=\Lambda \vec{P}(t) \\
l \vec{P}(\cdot)=\sum_{i=0}^{3} P_{i}(0)=1, P_{i}(0) \geq 0
\end{array}\right.
$$

In the development of work [9] Fig. 2 shows the kinetics of accumulation of cracks in the steering system of the tractors UMZ-8040.2 of different durations of operation with the allocation of the intensity of accumulation of small defects.

The coordinates of the graphs are as follows: ordinate axis $-n_{d} / N-$ the relative number of the detected cracks in the total number of investigated, significant from the point of view of safety of operation, details $N$; Axis abscissa $-t_{e k s} / t_{\text {base }}-$ ratio of the length of operation of tractors $t_{e k s}$ relative to the base duration $t_{\text {base }}=17$ years. The kinetics of the accumulation of cracks is described by the lines of the trend, the equations of which and the accuracy of approximation are shown in Fig. 2.

In this paper, the probabilistic distribution of changes in the states of the M-M-PE system was obtained for a traumatic situation that may occur due to malfunction of its steering system.

The following main reasons for the failure of the steering system of tractors were considered: presence of operational defects (cracks) in details that are detected by defectoscopic control; presence of backlashes, curvature of details and other violations of the system, which are visual inspection and testing; violation of the terms of technical inspection of tractors. 


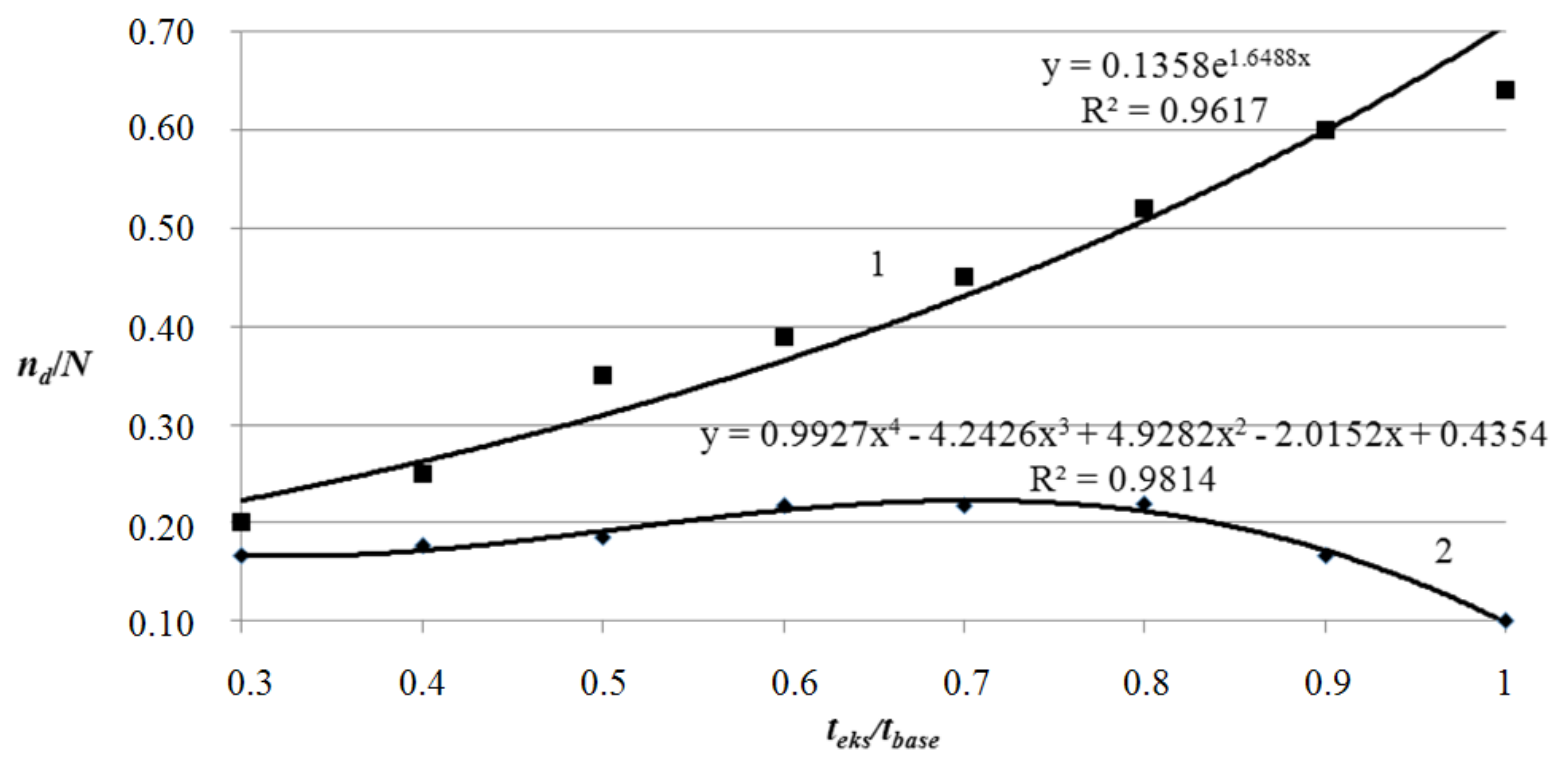

Fig. 2. Kinetics of accumulation of cracks in details of steering system of tractors of different durations of operation: 1 - cracks without spacing in size; 2 - small cracks

Elements of the matrix of the intensity of events (transition states of the system) were established on the basis of the following assumptions.

In the labor protection system for diagnosis of malfunctions of tractor units and other mobile agricultural machinery, Safety Ratings have been developed, which specify the frequency and place of control of the normative parameters of trouble-free operation of the machine nodes (units), but the emphasis is put on the visual (optical) control without the use of defectoscopic equipment.

This allows to detect significant discrepancy in the security parameters at the transitional stage. That is, non-compliance with the periodicity of such a control of the technical state will indicate the high probability of the system transition to a dangerous state.

In this paper, it was believed that the transition of the system from a normal state to a dangerous state (in relation to the safety of the machine operator), in addition to other circumstances related to the activities of the machine, may be due to the presence of a certain (critical) number of operational defects in a plurality of parts of the tractor units. That is, as a good condition of the system, the absence of operational defects or the presence of defects in the initial stages of their formation (that is, only small size), which in the near future will not lead to sudden failures (accidents) of the technology, were considered.

The failure of the system will be consistent with the absence of security equipment or cracking on the unit, which may subsequently overflow into the mains and cause the system failures (emergency situations). In the event, if the company will not take measures to repair the unit, the situation becomes dangerous (corresponding to the dangerous state of the system). It is possible to consider how dangerous a situation is (a dangerous state of the system), when the density of defects in an array of parts of the unit exceeds a certain critical value.

The number of tractors with a faulty steering system can be estimated from the data of 2018, when the mobile agricultural machinery held an annual state technical review. Thus, the number of tractors standing on the balance sheet of agricultural enterprises amounted to 190.375 units. Of these, 146.172 tractors were submitted for the state technical inspection. During the check, technical faults were detected in 33.383 tractors, which is $22.8 \%$.

As a result of the analysis of diagrams in Fig. 2, the following coefficients of intensity were proposed characterizing the intermediate states of the studied system:

1. We assume that at the initial stages of operation $\left(t_{e k s}=0.3\right)$ the intensity ratio will be $\lambda_{00}=1.00-$ $-0.22-0.16=0.62$, which corresponds to the proper condition of the tractors.

2. At the intermediate stage of operation $\left(t_{e k s}=0.5\right)$ the technical condition of tractors passes to the category conditionally serviceable due to the presence of details of operational defects 
$\left(\lambda_{01}=0.38\right)$. For tractors that are in the working condition (conditional condition), small cracks at present do not constitute a threat of sudden failure, the same $\lambda_{10}=0.22$. Then the working conditional state of the tractors will characterize the intensity coefficient $\lambda_{11}=1.00-0.38-$ $-0.22=0.40$.

3. For a faulty state, a significant accumulation of cracks of a critical (dangerous) size $\left(\lambda_{12}=\right.$ $=0.44-0.22=0.22)$ is characteristic for the maximum intensity of small cracks $\left(\lambda_{21}=0.22\right)$.

4. If the defectoscopic control of the responsible parts of the tractor is not carried out, the system at the exploitation phase $\left(t_{e k s}=0.9\right)$ will be dangerous for workers $\left(\lambda_{23}=0.6-0.17=0.43\right)$.

5. In the case of a technical inspection of tractors in accordance with the Safety Data Acquisition Control Maps, some parts of the tractor units will be found not to be in conformity with the safety requirements, these parts will be replaced and the state of the tractor will pass from the dangerous to the working state $\left(\lambda_{31}=0.228\right)$.

We will have a matrix of transition states of the system (4):

$$
\Lambda=\left(\begin{array}{llll}
-0.38 & 0.22 & 0 & 0 \\
0.38 & -0.44 & 0.22 & 0.228 \\
0 & 0.22 & -0.65 & 0 \\
0 & 0 & 0.43 & -0.228
\end{array}\right) .
$$

The set of probabilities of the states of the system can be obtained after solving the Kolmogorov equation with the condition of normalization (5):

$$
\left\{\begin{array}{c}
P_{0}(t)=0.907 c_{1}-0.302 c_{2}(0.477)^{t}-0.052 c_{3}(0.514) t-0.608 c_{4}(0.747)^{t} \\
P_{1}(t)=1.567 c_{1}+0.495 c_{2}(0.477)^{t}+0.067 c_{3}(0.514) t-0.243 c_{4}(0.747)^{t} \\
P_{2}(t)=0.53 c_{1}-1.194 c_{2}(0.477)^{t}-1.015 c_{3}(0.514) t-0.149 c_{4}(0.747)^{t} \\
P_{3}(t)=c_{1}+c_{2}(0.477)^{t}+c_{3}(0.514) t+c_{4}(0.747)^{t}
\end{array}\right.
$$

In order to fulfill the condition of valuation, it is necessary to put the constant $c_{2}=c_{3}=0 ; c_{1}=$ $=0.25 ; c_{4}=0.01$.

The functions of the states are represented in the following form (6):

$$
\left\{\begin{array}{c}
P_{0}(t)=-0.00608(0.747)^{t}+0.23 \\
P_{1}(t)=-0.00243(0.747)^{t}+0.39 \\
P_{2}(t)=-0.00149(0.747)^{t}+0.13 \\
P_{3}(t)=0.01(0.747)^{t}+0.25
\end{array}\right.
$$

The graphs of the calculated kinetic dependencies of probabilities $P$ states of the system are presented in Fig. 3.

The final probabilities of the states of the system will be: $P_{0}=0.23 ; P_{1}=0.39 ; P_{2}=0.13$; $P_{3}=0.25$. This means that in the long run (10 years), $23 \%$ of the units will work in the good condition $S_{0}, 39 \%$ and $13 \%$ will switch respectively to the defective $S_{1}$ and dangerous $S_{2}$ states, causing a dangerous situation to occur.

The probability of a system transitioning to a critical state of $S_{3}$ (traumatizing the machine) in this case will be $25 \%$. The probability of a system transitioning to a critical state of $S_{3}$ (traumatizing the machine) will be $25 \%$. 


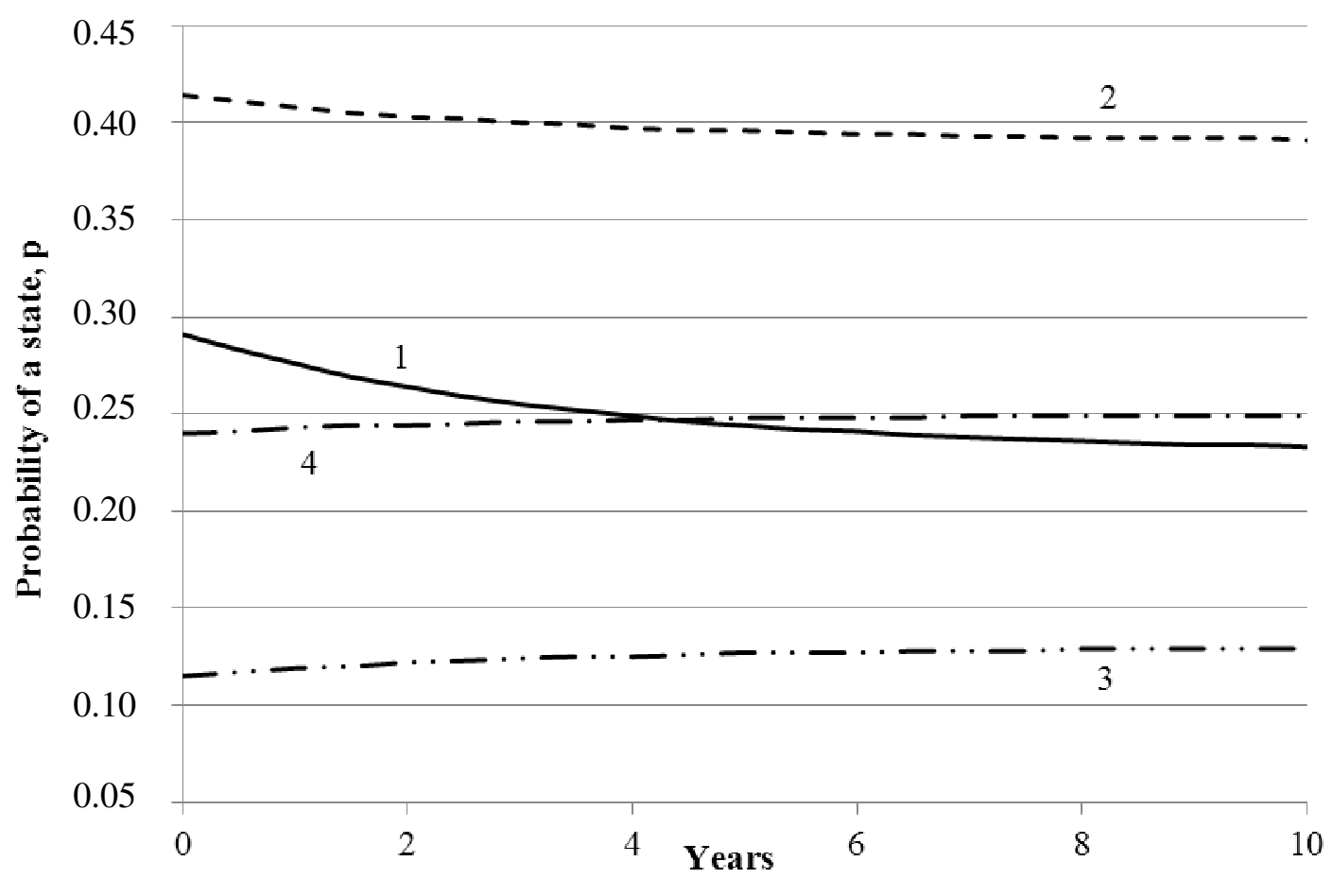

Fig. 3. Charts of kinetic dependences of probabilities $\mathbf{P}$ states of system: 1 - normal state $S_{0}$; 2 - conditional condition $S_{1} ; 3$ - faulty state $S_{3} ; 4$ - dangerous condition $S_{4}$

\section{Conclusions}

1. The method of investigation of changes in occupational risk at mechanized works in the agro industrial complex, taking into account the degree of accumulation of operational defect in an array of parts of MTA, is developed.

2. It was established that the processes of the course of traumatic situations and their consequences can be represented by graph structures, confined to four states of the system "man-machineindustrial environment": conditional, conditional and defective condition of the tractor (machine), entry of the machine operator into a dangerous (emergency) state (situation).

3. It is shown that the obtained results allow in the medium and long term to predict the probabilities of the states of the system "man-machine-industrial environment" in the categories of occupational risk of traumatizing agricultural mechanics.

\section{Acknowledgements}

This work was supported by a grant of the Ministry of Science and Education of Ukraine, contract _110/495/01.01.2016, Project code: UA 1649501 01, NULES Project title - "Experimental researches of parameters and modes of functioning of system of maintenance of agricultural machinery", phase 6: Experimental researches of parameters and modes of functioning of system of operational safety for agricultural aggregates.

\section{References}

[1] Ivanovs S., Bulgakov V., Nadykto V., Kuvachov V. Theoretical investigation of turning ability of two-machine sowing aggregate. Proceedings of 17th International Scientific Conference "Engineering for rural development". Jelgava, Latvia, May 23-25, 2018, Latvia University of Agriculture. Faculty of Engineering. Vol. 17, pp. 314-322.

[2] Bulgakov V., Pascuzzi S., Nadykto V., Ivanovs S. A mathematical model of the plane-parallel movement of an asymmetric machine and tractor aggregate. Journal of Agricultural Engineering. Vol. 49, No 1, 2018, pp. 258-271.

[3] Bulgakov V., Ivanovs S., Adamchuk V., Nowak J. Theoretical investigation of steering ability of movement of asymmetric swath headerand-tractor aggregate. Proceedings of 17th International 
Scientific Conference "Engineering for rural development". Jelgava, Latvia, May 23-25, 2018, Latvia University of Agriculture. Faculty of Engineering. Vol. 17, pp. 301-308.

[4] Knapczyk A., Francik S., Pedryc N., Hebda T. Bibliometric analysis of research trends in engineering for rural development. Proceedings of 17th International Scientific Conference "Engineering for rural development". Jelgava, Latvia, May 23-25, 2018, Latvia University of Agriculture. Faculty of Engineering. Vol. 17, pp. 700-707.

[5] Dubbini M., Pezzuolo A., De Giglio M., Gattelli M., Curzio L., Covi D., Yezekyan T., Marinello F. Last generation instrument for agriculture multispectral data collection. CIGR Journal, vol. 19, 2017, pp. 158-163.

[6] Viba J., Lavendelis E. Algorithm of synthesis of strongly non-linear mechanical systems. In Industrial Engineering - Innovation as Competitive Edge for SME, 22 April 2006. Tallinn, Estonia, pp. 95-98.

[7] Pinzi S., Cubero-Atienza A.J., Dorado M.P. Vibro-acoustic analysis procedures for the evaluationof the sound insulation characteristics of agricultural machinery. Journal of Sound and Vibration, vol. 266 (3), 2016, pp. 407-441.

[8] Yata V.K., Tiwari B.C., Ahmad, I. Nanoscience in food and agriculture: research, industries and patents. Environmental Chemistry Letters, vol. 16, 2018, pp. 79-84.

[9] Voinalovych A.V., Motrich M.N. Control of the technical state of agricultural aggregates by facilities of fault detection. Mechanization in agriculture. Sofia. Bulgaria, Year LXI, ISSN 08619638, issue 12, 2015, pp. 29-31. 